\title{
Wellens’ Syndrome and Impending Anterior Wall Myocardial Infarction: A Case Report
}

\section{Niniek Purwaningtyas* and Nuka Meriedlona}

Cardiology and Vascular Medicine, University of Sebelas Maret, Moewardi Hospital, Surakarta, Indonesia

*Corresponding author: Niniek Purwaningtyas, Cardiology and Vascular Medicine, University of Sebelas Maret, Moewardi Hospital, Surakarta, Indonesia, Tel: + 628122605006; E-mail: dr.niniek.spjp@gmail.com

Received Date: Nov 08, 2017; Accepted Date: Nov 25, 2017; Published Date: Dec 07, 2017

Copyright: (c) 2017 Purwaningtyas N, et al. This is an open-access article distributed under the terms of the Creative Commons Attribution License, which permits unrestricted use, distribution, and reproduction in any medium, provided the original author and source are credited.

\begin{abstract}
Wellens' Syndrome (WS) is a clinical presentation of ischemic chest pain with deep and symmetrical T wave or biphasic T wave inversion in V1 or V2 until V4 or V5 in electrocardiography finding and critical lesion in proximal Left Anterior Descenden (LAD) coronary artery. This case report discuss male 51 year old with typical chest pain and the ECG showed deep T-wave inversion in V1-V5. Transient ST elevation in V1-V5 appeared in 4th day of hospitalization without increasing cardiac markers. Coronary angiography examination demonstrated critical stenosis in proximal LAD $(90 \%)$. Here we discuss about Wellens' syndrome clinical manifestation, ECG and coronary angiography presentation, and also anterior wall Myocardial infarction as a consequences of WS. Early detection and prompt management can prevent anterior wall Ml and mortality.
\end{abstract}

Keywords: Wellens' syndrome; Proximal left anterior descenden

\section{Introduction}

Wellens' syndrome is a clinical manifestation of coronary artery disease developed as unstable angina pectoris or myocardial infarction without ST elevation. WS is characterized by typical chest pain with classic $\mathrm{T}$ wave inversion in precordial lead during chest pain and painfree period. Classic T wave inversion described as deeply T-wave or biphasic T-wave inversion. WS is known as LAD T-wave Syndrome indicated critical lesion in proximal LAD [1].

\section{Case Illustration}

A 51 year old male came to the emergency department with new onset of chest pain since 3 days before admitted. Chest pain was felt like heaviness and radiated to the back accompanied with cold sweating. Chest pain was progressively worsened especially in 10 hours before admitted to ED when he was working. Chest pain duration was 30 minute and did not relieve with rest. There is no nausea and vomiting. Patient had a chronic hypertension. Patient had no previous chest pain. History of diabetes mellitus and hyperlipidemia was denied. He had 20-pack-year smoking history and also alcohol intake.

From physical examination, vital signs were pulse: 70 beat per minute, blood pressure: $150 / 90 \mathrm{mmHg}$, and respiration rate: 20 times per minute. In cardiovascular examination within normal limit, there was no cardiomegaly, infiltrate, effusion and another abnormality on chest $\mathrm{x}$-ray. ECG showed normal rhythm sinus, normal axis, with heart rate 70 beat per minute, $\mathrm{LVH}$ cornell criteria and deep T-wave inversion in V1-V5 (Figure 1). There is no increasing of cardiac marker from laboratory finding. Echocardiography demonstrated normal Left Ventricular Ejection Fraction (LVEF) 60\% and global normokinetic of left ventricular wall. There was concentric left ventricular hypertrophy and diastolic dysfunction.

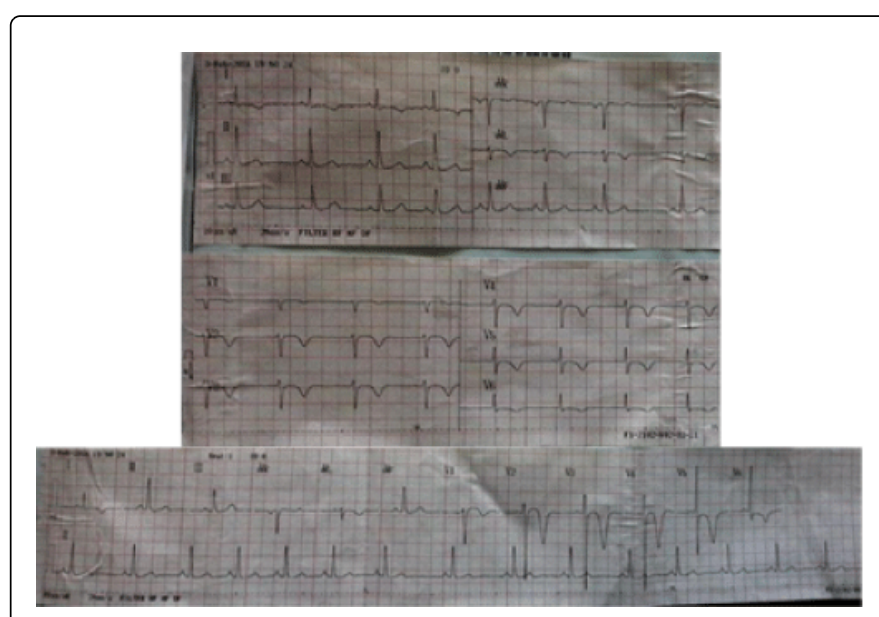

Figure 1: ECG in the emergency department (1st Day).

Aspirin $320 \mathrm{mg}$, Clopidogrel $300 \mathrm{mg}$, and Isosorbid dinitrat $5 \mathrm{mg}$ was administered in emergency department. The patient was admitted to the ICVCU and placed on anticoagulant fondaparinux $2.5 \mathrm{mg}$ daily subcutaneus injection, atorvastatin $40 \mathrm{mg}$, clopidogrel $75 \mathrm{mg}$, aspirin $80 \mathrm{mg}$, nitrat, bisoprolol $5 \mathrm{mg}$, and captopril $25 \mathrm{mg}$ three times daily for hypertension. Patient had severe chest pain in 4th day in the hospital. He also had emergency hypertension with blood pressure 220/120 mmHg, and heart rate was 72 beat per minute. The ECG showed ST elevation in V1-V5 and bigeminy premature ventricular contraction with normal value of cardiac marker (Figure 2). He was immediately given continuous nitroglyserin injection on syringe pump 20-40 mcg/minute amiodaron $150 \mathrm{mg}$ intravenously. After the blood pressure decreased to $150 / 90 \mathrm{mmHg}$, the chest pain was relived and ST elevation in the ECG was disappear and return to deep $\mathrm{T}$ wave inversion in V1-V5. 

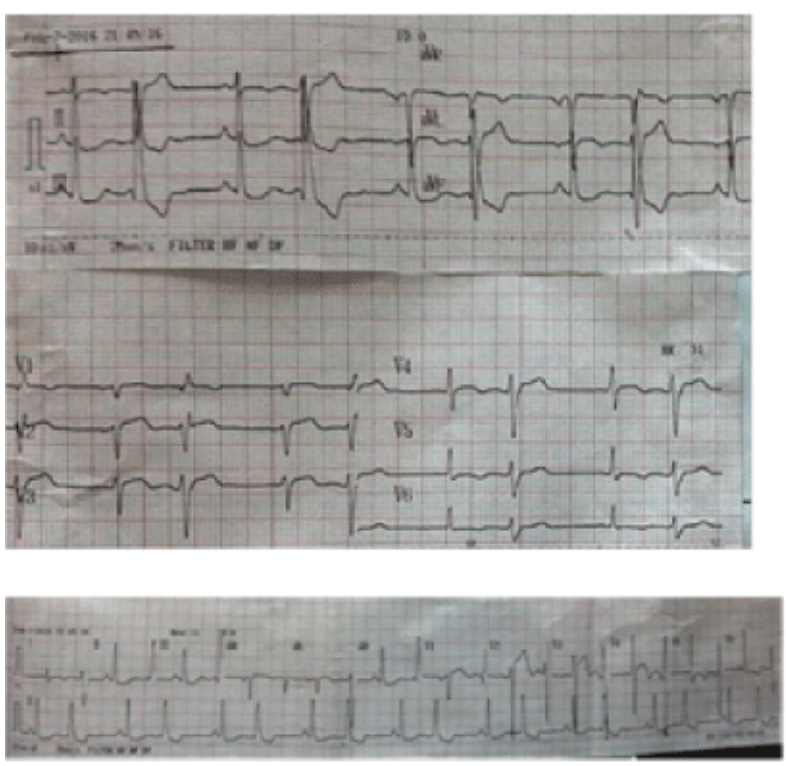

Figure 2: ECG of Transient ST Elevation (a) and 30 later (b) (4th day).

The patient was taken to the catheterization laboratory and coronary angiography was performed. The result was three vessel and left main disease with severe stenosis in proximal LAD 90\%. The patient was found to have $40 \%$ of stenosis in the mid-distal left main artery. Diffuse lesion was found in LAD and. Mild lesion was discovered in proximal left circumflexa $(50 \%)$ and mid right coronary artery (40-50\%) (Figure 3).
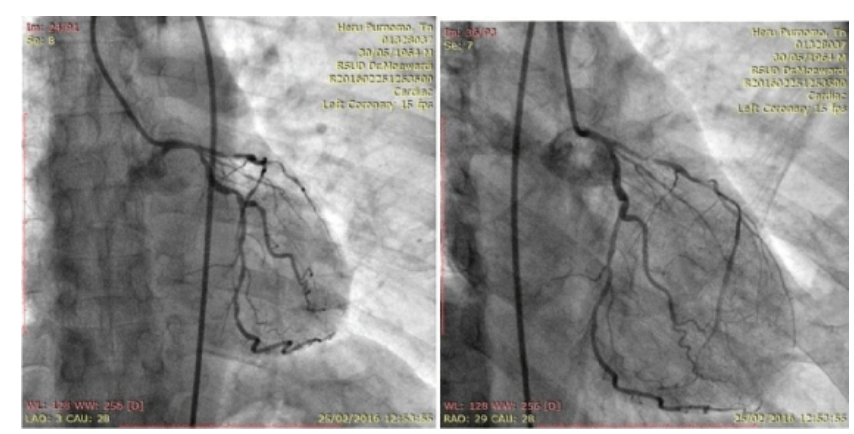

Figure 3: Coronary Angiography showed Critical Stenosis in Proximal LAD.

Based on history taking, physical examination, ECG, and coronary angiography finding, we diagnosed this patient as Type B Wellens' Syndrome with unstable angina pectoris and transient ST elevation manifestation as complication.
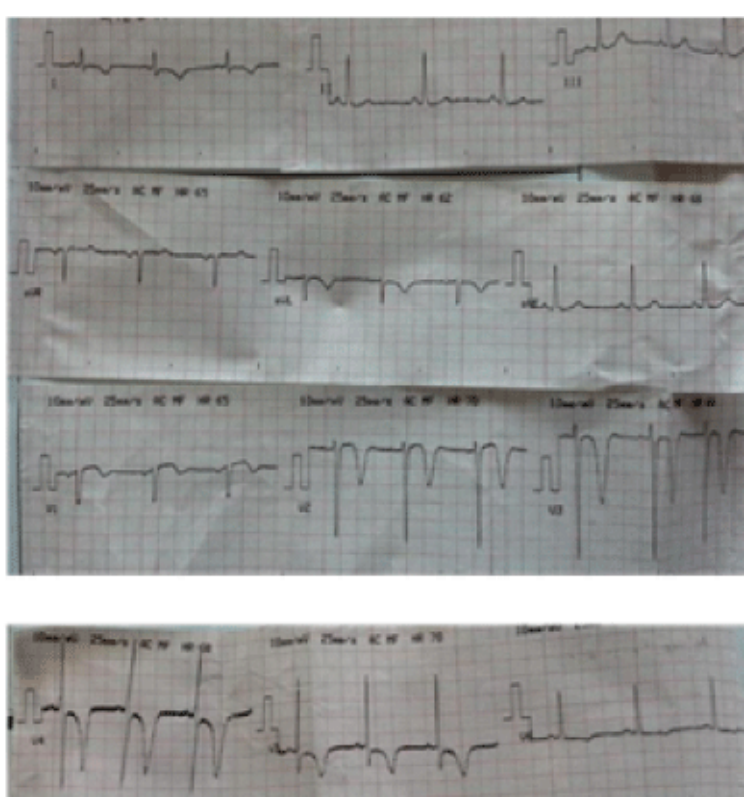

The patient was discharged and placed on atorvastatin, nitrat, captopril, bisopolol and dual antiplatelet (aspilet and clopidogrel) for outpatient therapy. He was referred to the cardiothoracic surgery for coronary artery bypass procedure in the following day.

\section{Discussion}

Wellens' syndrome was first described by de Zwaan C, Bar FW and Wellens' HJ in 1982. Their study from patients with unstable angina showed that $18 \%$ of subject had the wellenoid ECG pattern and the results was $75 \%$ of the subject who did not performed revascularization developed anterior wall myocardial infarction within a few weeks [2]. The second study in 1989, from all patients with Wellens' syndrome, ECG patterns were found significant stenosis in proximal LAD artery [3].

WS consist of typical chest pain as clinical manifestation and classic T-wave inversion in ECG finding suggesting for severe stenosis in proximal LAD coronary artery [4]. Critical stenosis in LAD artery had a severe complication and called as widow maker lesion. LAD origin was from left main coronary artery, supplies the anterior myocardial through interventricular sulcus. Occlusion in LAD can cause severe left ventricular dysfunction and increase risk of congestive heart failure and mortality. WS was diagnosed based on classic T-wave inversion in ECG finding during chest pain and pain free [5]. 


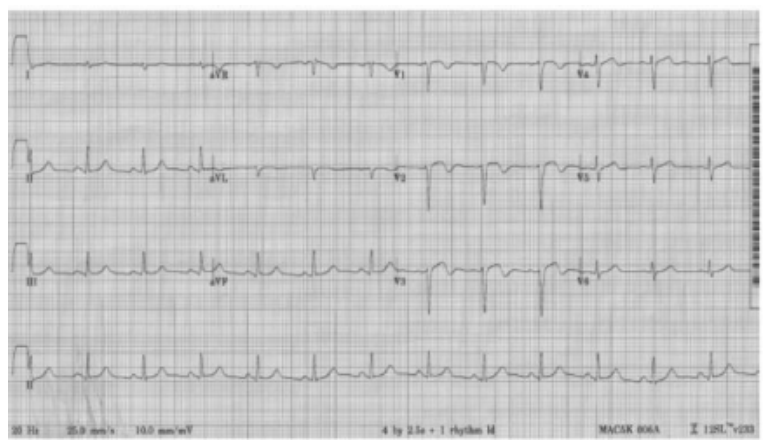

Figure 4: Wellens' Syndrome ECG's Pattern. Type A (a) and Type B (b).

Study from Mao Liu in 2016 from 275 unstable angina patients, 35 patients had wellenoid T-wave ECG pattern in V2-V3. It predicts LAD artery stenosis with $18.92 \%$ of sensitivity, $96.67 \%$ specificity, and $92.11 \%$ of predictive value [6].

Wellens' syndrome was divided into two variant based on ECG finding, type A was biphasic T-wave inversion and type B was deep T-

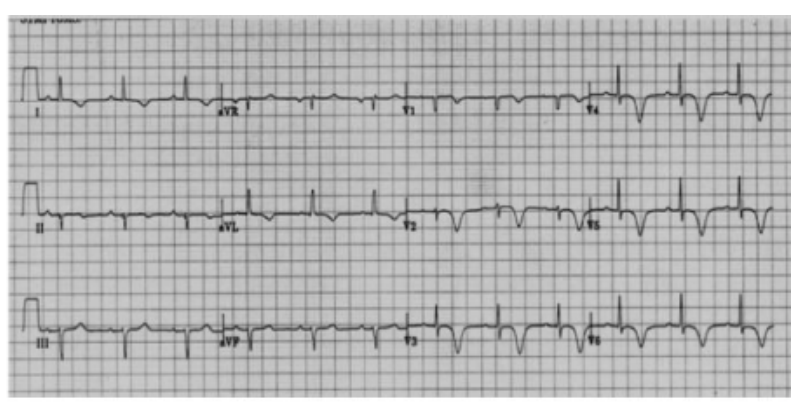

wave inversion in precordial lead V1 or V2 until V4 or V5 (Figure 4). Type B is more common, occurring in $76 \%$ cases, type A occurs in $24 \%$ cases. These T-waves changes represent reperfusion of the myocardium (Table 1) [4].

\section{Clinical Manifestation and ECG criteria of WS [7]}

Biphasic or deep T-wave inversion in precordial lead V2 or V3 until V4 or V5

Cardiac marker is normal or slightly increasing

Minimal ST elevation (less than $1 \mathrm{~mm}$ ) or no ST elevation

No poor $\mathrm{R}$ wave progression and Pathologic $\mathrm{Q}$ wave

History of angina

Table 1: Clinical manifestation and ECG criteria of WS.

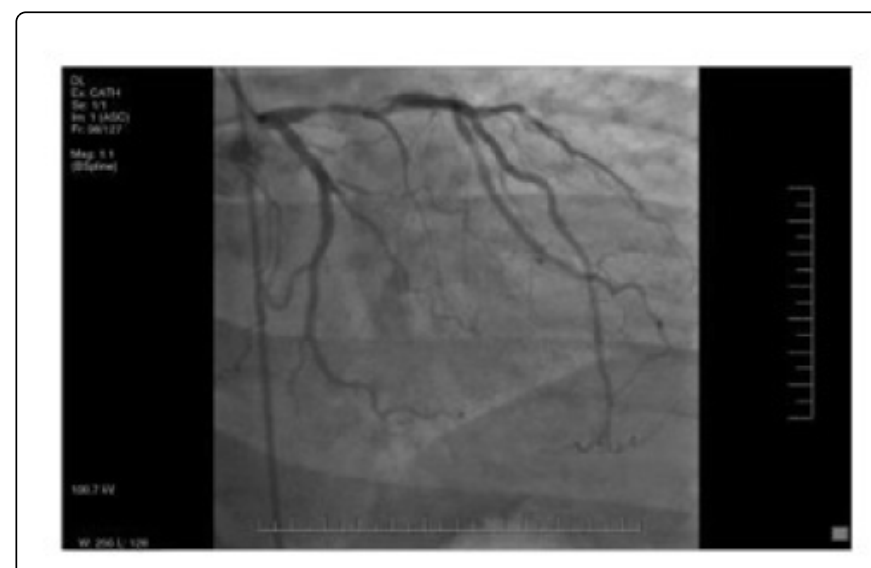

Figure 5: Coronary angiography of critical lesion in Proximal LAD coronary artery $[8]$.

A case report of Wellens' Syndrome from Hollar et al. in 2015, had shown critical stenosis of proximal LAD $90 \%$ from urgent cardiac catheterization (Figure 5). Those patients had an unstable angina as clinical manifestation. Recognition of ECG finding appropriately was important for urgent catheterization diagnostic and management of revascularization. Progressive complication of anterior wall myocardial infarction could be prevented [8].

Wellens' syndrome was early sign of pre-infarction LAD stenosis. Treadmill Stress Test should be avoided because it potentially induced myocardial infarct and also results in severe left ventricular dysfunction. Prediction of severity of coronary artery from ECG is very important for decreasing morbidity and mortality of Wellens' syndrome [3]. Early management of Wellens' syndrome is generally same as acute coronary syndrome (Table 2). Patient had to be transferred to nearest hospital and if needed with capable urgent catheterization. Serial ECG and laboratory of cardiac enzyme should be performed. Dual antiplatelet and anti-angina as soon as possible should be administered in emergency department. When WS diagnosis was established, coronary angiography should be performed. It is useful for evaluating further management, angioplasty or coronary artery bypass procedure [4]. 
Citation: Purwaningtyas N, Meriedlona N (2017) Wellens' Syndrome and Impending Anterior Wall Myocardial Infarction: A Case Report. Med Rep Case Stud 2: 145. doi:10.4172/2572-5130.1000145

Page 4 of 4

\begin{tabular}{|l|}
\hline Early Management of Wellens' syndrome \\
\hline Reffered to the nearest hospital \\
\hline Maintenance airway, breathing and circulation \\
\hline Oxygen supplement and monitoring of vital sign, intravenous acces, aspirin, clopidogrel, nitrat, beta blocker, and morphine if needed \\
\hline Serial ECG examination \\
\hline Laboratory examination included cardiac marker (troponin I or T) \\
\hline Chest X-ray \\
\hline
\end{tabular}

Table 2: Early management of Wellens' syndrome [3].

\section{Conclusion}

Early recognizing from electrocardiography pattern of Wellens Syndrome can predict critical stenosis in proximal LAD. WS is susceptible developed into ST Elevation myocardial infarction. Early detection and prompt management is very important to prevent complication and mortality. Coronary angiography had to be immediately performed for further management, angioplasty or coronary artery bypass surgery. Optimal medical therapy is still needed for relieve symptoms and avoid deterioration of stenosis.

\section{References}

1. Goldberger AL (2012) Clinical electrocardiography: a simplified approach. Elsevier Health Sciences 8: 89-91.

2. De Zwaan C, Bar FW, Wellen HJ (1982) Characteristic electrocardiography pattern indicating a critical stenosis high in left anterior descending coronary artery in patients admitted because of impending myocardial infarction. Am Heart J 103: 730-736.
3. Mead NE, O'Keefe KP (2009) Wellen's syndrome: An ominous EKG pattern. J Emerg Trauma Shock 2: 206-208.

4. Kardesoglu E, Celik T, Cebeci BS, Cingozbay BY, Dincturk M, et al. (2003) Wellen's Syndrome: A Case Report. J Int Med Res 31: 585-590.

5. Muharam MY, Ahmad R, Harmy MY (2012) The 'widow maker': Electrocardiogram features that should not be missed. Malays Fam Physician 8: 45-47.

6. Liu M, Han C, Ke J, Tang W, Tan G, et al. (2016) Wellenoid T-Wave is an Important Indicator for severe coronary Artery stenosis. Int J Cardiol 209: 22-23.

7. Balasubramanian K, Balasubramanian R, Subramanian A (2013) A Dangerous twist of the ' $T$ ' wave: A case of Wellen's Syndrome. Australas Med J 6: 122-125.

8. Hollar L, Hartness O, Doering T (2015) Recognizing Wellens' syndrome, a warning sign of critical proximal LAD artery stenosis and impending anterior myocardial infarction. J Community Hosp Intern Med Perspect 